EDUCATION (IMAGE)

OFFICIAL JOURNAL

MJPCH Vol. 26 (2) December 2020

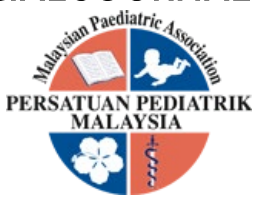

\title{
FLOW CYTOMETRIC ASSESSMENT OF X-LINKED AGAMMAGLOBULINEMIA
}

\author{
Zarina Thasneem Zainudeen, Ilie Fadzilah Hashim, Intan Juliana Abd Hamid
}

Received: 21 August 2020; Accepted revised manuscript: 20 October 2020

Published online: 17 November 2020

X-Linked Agammaglobulinemia (XLA) is the most common form of humoral immunodeficiency and is caused by mutations in the BTK gene, which encodes Bruton's tyrosine kinase (BTK), a hematopoietic-specific tyrosine kinase. The congenital defect occurs in B-lymphopoiesis, resulting to an arrest of pre-B cells into later $B$-cell stages, or incomplete differentiation of B-cell precursors to pre-B cells. X-linked agammaglobulinemia is characterized by $a$ significant reduction or absence of circulating $B$ cells in blood and profound hypogammaglobulinemia of all isotypes $(\lg G, \lg A$, $\lg \mathrm{M}$, and $\lg \mathrm{E}$ ) in affected males[1]. The clinical presentation includes early onset recurrent and severe bacterial infections, more often after the age of 2 years[2].

Majority of BTK mutations are observed to be associated with a reduction in BTK MRNA and BTK protein[3]. The product of the BTK gene has been detected in B cells and other hematopoietic cells, such as platelets and monocytes[4]. Since XLA patients are lacking circulating $B$ cells, the detection of BTK expression in non-B cells can be clinically informative to detect the deficiency, even in carriers[5].
Intracellular BTK staining of monocytes was performed by labelling peripheral blood with CD14 FITC, followed by the fixation, permeabilization and labelling with anti-BTK Alexa Fluor 647 monoclonal antibodies (MoAbs) and Alexa Fluor 647 Mouse lgG2a isotype control for 30-60 minutes at room temperature. Mononuclear cells were stained before cellular permeabilization to allow the detection of monocytes from other cells. The stained cells were washed and prepared for flow cytometric analysis. All the patients were also analyzed for lymphocyte sub-population by staining whole blood with MoAbs for CD3, CD4, CD8, CD19, CD20 and CD16 (Lyse/wash method).

\footnotetext{
Primary Immunodeficiency Diseases Group, Cluster of Regenerative Medicine, Advanced Medical and Dental Institute (AMDI), Universiti Sains Malaysia, Penang.

Corresponding author: Zarina Thasneem Zainudeen, Advanced Medical and Dental Institute (AMDI), SAINS@Bertam, Universiti Sains Malaysia, 13200, Kepala Batas, Penang, Malaysia.

Tel: +604-5622175 Email: zarina.zainudeen@usm.my
} 
A

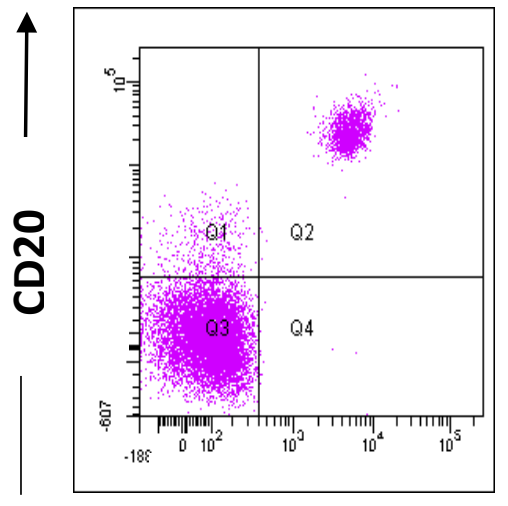

Normal

Patient

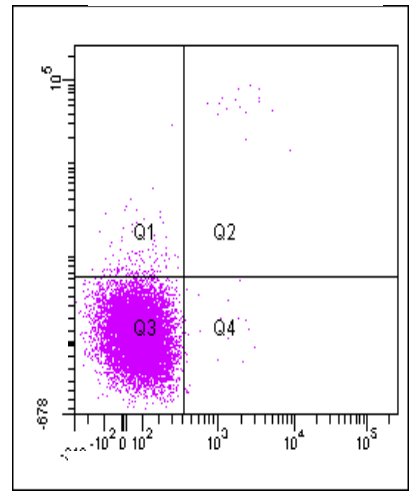

CD19

B

Normal

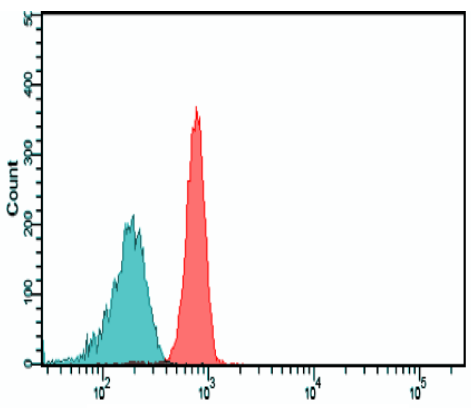

Patient

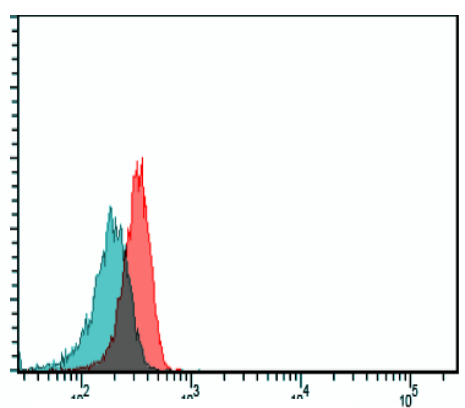

Mother

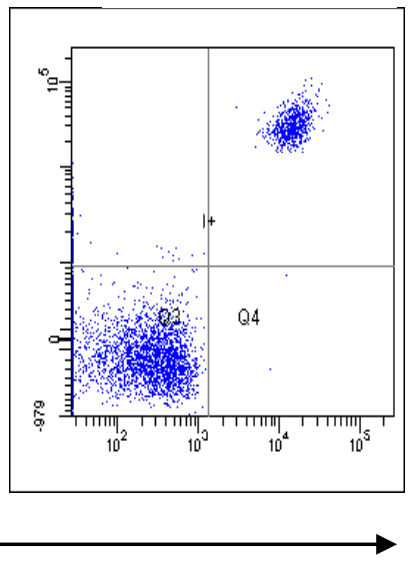

Mother

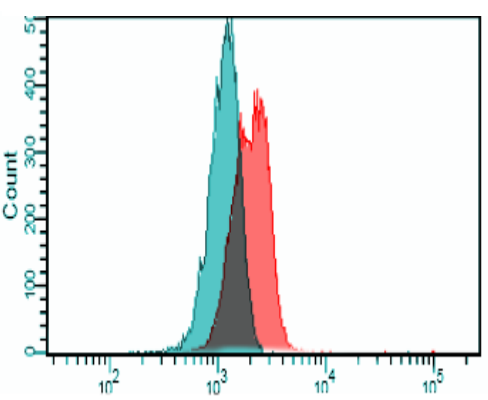

\section{BTK}

Figure 1: Results of our flow cytometry-based assay of BTK expression in a normal donor, XLA patient and the mother. (A) FACS dot plot of CD20 and CD19 gated for lymphocytes. B cells (CD19 and CD20) were markedly decreased in the blood of the patient while the mother possessed a substantial number of circulating $B$ cells (B) Histograms gated on CD14+ monocytes expressing BTK. Blue shaded area indicates the control antibody. The defective BTK expression in monocytes was seen in XLA patient and a bimodal pattern of normal and abnormal BTK expression was also observed in his mother, known as an obligate carrier. The father follows a pattern as the normal donor (result not shown). 


\section{Conclusion}

Our report concludes that the family study of Bruton's agammaglobulinemia indicated an $X$ linked inheritance. The bimodal BTK expression in monocytes is very helpful in carrier detection. Because of the rapidity of this assessment, it is currently advised that a hypoammaglobulinemic male with a lack of circulating B cells should be initially examined by flow cytometric assay. Even though it is helpful to correlate gene and protein data with clinical history, but in a situation with limited resources and access to genetic tests, flow cytometric analysis is beneficial in making a final diagnosis of XLA.

\section{Disclaimer}

This assay was analyzed on BD FACS Canto II at Advanced Medical and Dental Institute, USM, Penang.

\section{Acknowledgment}

The authors wish to acknowledge USM for the financial and moral support in PID service empowerment through the USM 2020 Initiatives (Project 19) (USM: 311.CIPPT.411919).

\section{References:}

[1.] Kanegane $H$, Futatani $T$, Wang $Y$, Nomura $K$, Shinozaki $\mathrm{K}$, Matsukura $\mathrm{H}$, et al. Clinical and mutational characteristics of X-linked agammaglobulinemia and its carrier identified by flow cytometric assessment combined with genetic analysis. J Allergy Clin Immunol. 2001;

[2.] Aghamohammadi $A$, Parvaneh $N$, Kanegana $H$, Moin $M$, Amirzargar AA, Farhoudi $A$, et al. Screening of the Bruton Tyrosine Kinase (BTK) Gene Mutations in 13 Iranian Patients with Presumed X-Linked Agammaglobulinemia. Iran J Allergy Asthma Immunol. 2004;

[3.] Futatani T, Miyawaki T, Tsukada S, Hashimoto S, Kunikata T, Arai S, et al. Deficient expression of Bruton's tyrosine kinase in monocytes from $\mathrm{X}$ - linked agammaglobulinemia as evaluated by a flow cytometric analysis and its clinical application to carrier detection. Blood. 1998;

[4.] Tsukada S, Saffran DC, Rawlings DJ, Parolini O, Allen RC, Klisak I, et al. Deficient expression of a B cell cytoplasmic tyrosine kinase in human X-linked agammaglobulinemia. Cell. 1993;

[5.] Ochs HD, Smith CIE. X-linked agammaglobulinemia: $A$ clinical and molecular analysis. Medicine. 1996. 